\title{
Origin of high oscillator strength in green-emitting InGaN/GaN nanocolumns
}

\section{AUTHOR(S):}

Kawakami, Y; Suzuki, S; Kaneta, A; Funato, M; Kikuchi, A; Kishino, K

\section{CITATION:}

Kawakami, Y ...[et al]. Origin of high oscillator strength in green-emitting InGaN/GaN nanocolumns. APPLIED PHYSICS LETTERS 2006, 89(16): 163124.

\section{ISSUE DATE:}

2006-10-16

URL:

http://hdl.handle.net/2433/50151

\section{RIGHT:}

Copyright 2006 American Institute of Physics. This article may be downloaded for personal use only. Any other use requires prior permission of the author and the American Institute of Physics. 


\title{
Origin of high oscillator strength in green-emitting InGaN/GaN nanocolumns
}

\author{
Y. Kawakami, ${ }^{\text {a) }}$ S. Suzuki, A. Kaneta, and M. Funato \\ Department of Electronic Science and Engineering, Kyoto University, Kyoto 615-8510, Japan
}

\author{
A. Kikuchi and K. Kishino \\ Department of Electrical and Electronics Engineering, Sophia University, Tokyo 102-8554, Japan
}

(Received 19 July 2006; accepted 31 August 2006; published online 20 October 2006)

\begin{abstract}
Optical characterization has been performed on an InGaN/GaN nanocolumn structure grown by nitrogen plasma assisted molecular beam epitaxy not only in macroscopic configuration but also in a microscopic one that can be assessed to a single nanocolumn. The photoluminescence (PL) decay monitored at $500 \mathrm{~nm}$ is fitted with a double exponential curve, which has lifetimes of 0.67 and $4.33 \mathrm{~ns}$ at $13 \mathrm{~K}$. These values are two orders of magnitude smaller than those taken at the same wavelength in conventional InGaN/GaN quantum wells (QWs) grown toward the C orientation. PL detection of each single nanocolumn was achieved using a mechanical lift-off technique. The results indicate that the very broad, macroscopically observed PL spectrum is due to the sum of the sharp PL spectrum from each nanocolumn, the peak energy of which fluctuates. Moreover, unlike conventional QWs, the blueshift of a single nanocolumn is negligibly small under higher photoexcitation. These findings suggest that carrier localization as well as the piezoelectric polarization field is suppressed in InGaN/GaN nanocolumns. (c) 2006 American Institute of Physics. [DOI: $10.1063 / 1.2363958]$
\end{abstract}

In recent years, the developments in the growth technology of GaN-based semiconductors have led to the commercialization of dazzling blue light emitting diodes (LEDs) and violet laser diodes (LDs). Although the emission wavelength can be controlled from ultraviolet $(360 \mathrm{~nm})$ to violet $(400-420 \mathrm{~nm})$, blue $(440-470 \mathrm{~nm})$, green $(500-540 \mathrm{~nm})$, and amber $(580-600 \mathrm{~nm})$ regions by increasing the In mole fraction in the $\operatorname{In}_{x} \mathrm{Ga}_{1-x} \mathrm{~N}$ active layer, device performance rapidly degrades if the wavelength is beyond the blue-green spectral range. Consequently, the practical external quantum efficiency of green LEDs is about half of that of blue LEDs, and the longest operational LD wavelength is about $480 \mathrm{~nm}$.

These green gap problems are probably due to two major factors. The first one is exciton/carrier localization induced by random potential fluctuation and the second one is the quantum confinement Stark effect (QCSE) caused by strong piezoelectric and spontaneous polarization in strained $\mathrm{InGaN} / \mathrm{GaN}$ quantum wells (QWs). The former improves the internal efficiency $\left(\eta_{\text {int }}\right)$ by suppressing the pathway of nonradiative processes, while the compositional modulation coupled with the dislocations may generate additional nonradiative recombination centers. However, the latter degrades the $\eta_{\text {int }}$ by reducing the oscillator strength of the excitons in the well, which is inversely proportional to the radiative recombination lifetime, because the overlap between the electron and hole wave functions perpendicular to the growth direction is lowered by the QCSE. Therefore, it is important to assess and modify carrier/exciton recombination processes so that positive feedback can be made to the growth conditions and to the appropriate design of the device structures.

Consequently, InGaN/GaN QWs grown on GaN nanocolumns (InGaN/GaN nanocolumns) have been proposed ${ }^{1,2}$ as promising emitters due to the very low density of the threading dislocations compared to that in conventional C-oriented InGaN/GaN QW films. Moreover, the typical diameter of a nanocolumn ${ }^{1-5}$ is between a few tens of nanom-

${ }^{a)}$ Electronic mail: kawakami@kuee.kyoto-u.ac.jp eters to a few hundreds of nanometers. Thus, the size of a nanocolumn can be less than the diffusion length of the photogenerated carriers (and/or excitons) in conventional samples, which leads to a reduction in the localization effect. In this letter, detailed optical properties in a sample of InGaN/GaN nanocolumns grown on (0001)-oriented sapphire are assessed using photoluminescence (PL) spectroscopy.

The nanocolumn structure of the $\mathrm{InGaN} / \mathrm{GaN}$ single QW (SQW) assessed in this letter was grown by molecular beam epitaxy with a nitrogen-plasma source on a (0001)oriented sapphire substrate. The layer is composed of a $1.1 \mu \mathrm{m}$ thick GaN buffer layer, a $3 \mathrm{~nm}$ InGaN SQW, and a $55 \mathrm{~nm} \mathrm{GaN}$ capping layer. The diameter of the nanocolumns as determined by scanning electron microscopy ranged between 40 and $120 \mathrm{~nm}$ and had a mean value of approximately $80 \mathrm{~nm}$. Entire layers were undoped and the background donor concentration was less than $1 \times 10^{17} \mathrm{~cm}^{-3}$.

Figure 1 shows the macroscopic PL spectra from a sample taken at (a) $13 \mathrm{~K}$ and (b) room temperature (RT) under continuous wave excitation with a $\mathrm{He}-\mathrm{Cd}$ laser $(325 \mathrm{~nm})$. The estimated number of photoexcited nanocolumns is about a few hundred thousands due to the area of photoexcitation (100 $\mu \mathrm{m}$ in diameter). The temperature dependence of the PL intensity shows that nonradiative recombination processes are negligible below $50 \mathrm{~K}$, where estimation of internal quantum efficiency is based on the assumption where the efficiency at cryogenic temperature is nearly equal to $100 \%$. The validity of this was checked by means of thermal lens spectroscopy, ${ }^{6}$ by which the heat generation due to nonradiative recombination was found to be negligible processes in the nanocolumn samples at low temperatures. Thus, the estimated $\eta_{\text {int }}$ value of integrated PL intensity is $12 \%$ at RT. The peak wavelength is located near $512 \mathrm{~nm}$ at $13 \mathrm{~K}(539 \mathrm{~nm}$ at $\mathrm{RT})$, which corresponds to a green color. However, the PL linewidth is as large as $0.56 \mathrm{eV}$ at $13 \mathrm{~K}(0.45 \mathrm{eV}$ at $\mathrm{RT})$, so that the spectrum covers not only the red $(600 \mathrm{~nm})$ component, but the blue $(450 \mathrm{~nm})$ one as 


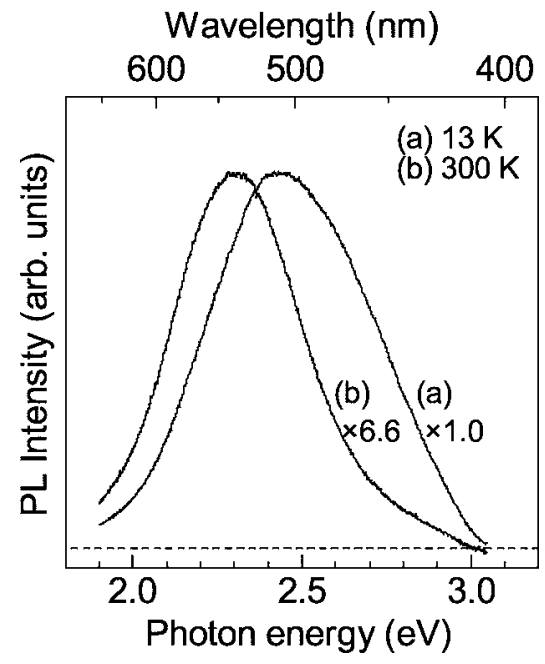

FIG. 1. PL spectra taken at InGaN/GaN nanocolumns at (a) $13 \mathrm{~K}$ and at (b) RT under macroscopic photoexcitation. The internal quantum efficiency of integrated PL intensity is $12 \%$ at RT.

well. The observed PL linewidths are fairly large compared to that observed in a conventional green light emitting InGaN/GaN SQW film that is on the order of $0.1 \mathrm{eV}$. It is noted that the temperature dependence of the PL linewidth is opposite to that in conventional semiconductors, where it becomes broader with increasing temperature. This is probably because the thermal quenching of the shorter wavelength component is more dominant in the nanocolumn sample. This is also consistent with a large redshift of peak energy $(0.12 \mathrm{eV})$ between $13 \mathrm{~K}$ and RT, which is almost twice as large as that in GaN. It is difficult to reveal the origin of nonradiative recombination centers in nanocolumn samples. However, the contribution of surface states may play an important role for the nonradiative recombination centers. One possibility is that energy levels contributing to shorter wavelength can transfer more easily to the surface trapping states through the resonant process. However, this is still a speculation, so that further study is needed for detailed understanding. In order to assess the spatial distribution of the emission, fluorescent images were observed at RT with an optical microscope under $\mathrm{Hg}$-lamp excitation $(365 \mathrm{~nm})$ as shown in Fig. 2. The images consist of numerous bright spots, which are mostly green, but some are blue, yellow, and red. Although the spatial resolution of the microscope is insufficient to resolve individual nanocolumns, the images suggest that the very broad PL is not solely due to the inhomo-

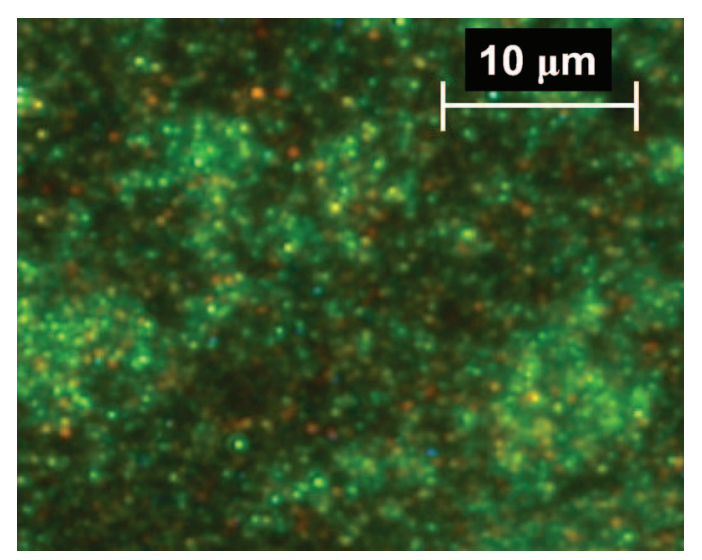

FIG. 2. (Color) Fluorescent microimage taken at $\mathrm{InGaN} / \mathrm{GaN}$ nanocolumns at RT under photoexcitation of the $365 \mathrm{~nm}$ line from a $\mathrm{Hg}$ lamp. at RT under photoexcitation of the $365 \mathrm{~nm}$ line from a Hg lamp. mechanical scriber technique. The removed nanocolumns
Downloaded 05 Mar 2008 to 130.54 .110 .22 . Redistribution subject to AIP license or copyright; see http://apl.aip.org/apl/copyright.jsp
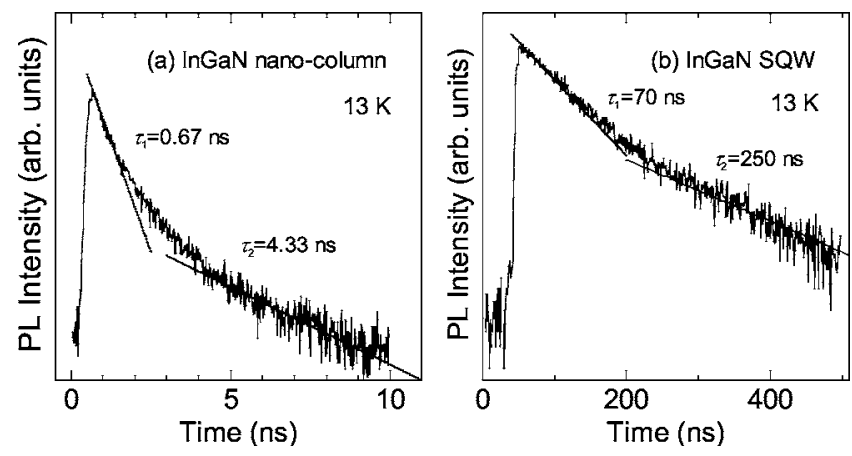

FIG. 3. PL decay curves of the $500 \mathrm{~nm}$ emission band taken at (a) InGaN/GaN nanocolumns and (b) an InGaN/GaN SQW film at $13 \mathrm{~K}$.

geneous broadening within each nanocolumn, but is due to the sum of the spectrum from each nanocolumn.

Time-resolved PL spectroscopy has also been performed on the sample at $13 \mathrm{~K}$. The PL decay $[I(t)]$ is fitted well with double exponential curves that have fast $\left(\tau_{1}\right)$ and slow $\left(\tau_{2}\right)$ components, where $I(t)=A_{1} \exp \left(-t / \tau_{1}\right)+A_{2} \exp \left(-t / \tau_{2}\right)$. It was found that the recombination process is dominated by the fast component because the value of $A_{1} / A_{2}$ is between 3 and 6 in the full range of the monitoring wavelength. It is noteworthy that the values of $\tau_{1}$ and $\tau_{2}$ are about 0.7 and $4 \mathrm{~ns}$, respectively, and are nearly independent of the monitoring wavelength between 450 and $600 \mathrm{~nm}$. This observation is different from the system observed in the recombination dynamics of mobile localized excitons, where the PL lifetimes decrease as the wavelength decreases in the shortwavelength tail of the spectra, and supports the finding that the PL spectra are composed of those from individual nanocolumns. Nevertheless, the observed recombination lifetimes and the spectral dependence of $A_{1} / A_{2}$ are valuable for studying how the dimensionality of excitons ${ }^{7,8}$ is distributed in each nanocolumn. Analysis of the exciton dimensionality is currently in progress. Another interesting feature is the very rapid radiative lifetimes compared to those in InGaN SQWs conventionally grown toward $\mathrm{C}$ orientation. Figure 3 plots the decay curve of the PL intensity monitored at $500 \mathrm{~nm}$ after a pulsed excitation for (a) the InGaN/GaN nanocolumn and (b) the conventional InGaN/GaN SQW. The well layer thickness for both samples was $3 \mathrm{~nm}$. For the conventional sample, the decay curve can be fitted by the parameters of $\tau_{1}=70 \mathrm{~ns}, \tau_{2}=250 \mathrm{~ns}$, and $A_{1} / A_{2}=4.5$ under a moderately low photoexcitation density. These lifetimes are two orders of magnitude larger than that of a violet $(400 \mathrm{~nm})$ emitting sample, having a similar structure except for the lower In mole fraction in the InGaN well. This effect is mainly due to the QCSE where wave functions of electrons and holes in the well are separated due to a piezoelectric field, which leads to a reduced oscillator strength and redshifted transition energies. Because the screening effect due to injected carriers lowers the electric fields, an increased oscillator strength and blueshift are observed under higher photoexcitation (or current injection). On the other hand, the PL decay of an $\mathrm{InGaN} / \mathrm{GaN}$ nanocolumn monitored at $500 \mathrm{~nm}$ is expressed by a double exponential curve with parameters of $\tau_{1}$ $=0.67 \mathrm{~ns}, \tau_{2}=4.33 \mathrm{~ns}$, and $A_{1} / A_{2}=5.9$, which suggests that the internal field is suppressed.

In order to assess the PL spectra of individual nanocolumns, a deposited array of columns was removed by the 


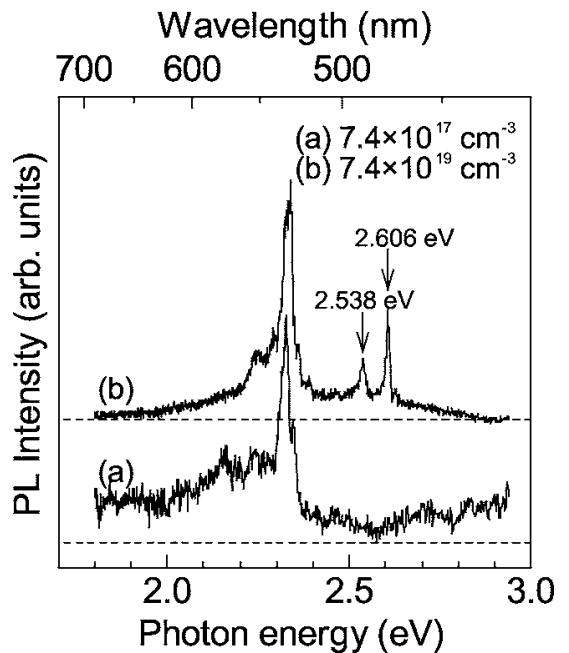

FIG. 4. PL spectra taken at a single $\mathrm{InGaN} / \mathrm{GaN}$ nanocolumn at $13 \mathrm{~K}$ under (a) $I_{\mathrm{ex}}=6.8 \mu \mathrm{J} / \mathrm{cm}^{2}$ and (b) $I_{\mathrm{ex}}=680 \mu \mathrm{J} / \mathrm{cm}^{2}$.

were then dispersed in ethanol and the solution was dropped onto a quartz plate. After drying, each column was separately dispersed on a plate. In this measurement, the PL spectra were monitored through an optical microscope by siteselective photoexcitation of each nanocolumn. Figure 4(a) shows the PL spectrum of a single nanocolumn under a photoexcitation energy density $\left(I_{\mathrm{ex}}\right)$ of $6.8 \mu \mathrm{J} / \mathrm{cm}^{2}$ at $13 \mathrm{~K}$. For this measurement, a frequency-doubled $\mathrm{Al}_{2} \mathrm{O}_{3}$ :Ti laser was used to selectively excite the well when the operation conditions included a wavelength of $400 \mathrm{~nm}$, a pulse width of $2 \mathrm{ps}$, and a repetition rate of $80 \mathrm{MHz}$. The obtained PL signal was integrated within the entire time domain. Considering the absorbance in the well, the number of photogenerated carriers (excitons) $\left(N_{e}\right)$ is approximately $7 \times 10^{17} \mathrm{~cm}^{-3}$. The main PL peak is located near $2.339 \mathrm{eV}$. A few tens of single nanocolumns were tested, and it was found that the PL peak energies are scattered from 2.2 to $2.6 \mathrm{eV}$, which clearly reveals that the broad macroscopic PL is due to the fluctuation of the PL peaks from individual nanocolumns. The PL linewidth in Fig. 4(a) is about $20 \mathrm{meV}$ and is mainly due to alloy broadening, which is inevitable because of the standard deviation of the alloy composition within the exciton Bohr radius (about $3 \mathrm{~nm}$ in $\mathrm{In}_{x} \mathrm{Ga}_{1-x} \mathrm{~N}$ wells). ${ }^{9}$

Figure 4(b) shows a PL spectrum under higher photoexcitation $\left(I_{\mathrm{ex}}=680 \mu \mathrm{J} / \mathrm{cm}^{2}\right.$, and $\left.N_{e}=7 \times 10^{19} \mathrm{~cm}^{-3}\right)$. Although the main PL band slightly broadens, the blueshift of peak energy is as small as $5 \mathrm{meV}$. This shift is much smaller than that observed in a conventional InGaN/GaN SQW film emitting at $500 \mathrm{~nm}$ because a conventional film is blueshifted by approximately $100 \mathrm{meV}$ under the same photopumping conditions due to the screening of the piezoelectric field and the band filling of the localized states. The very small blueshift observed in a single nanocolumn shows that both these ef- fects are suppressed by the mechanism discussed below. The reduction in the localization effect is probably because the size of the nanocolumn is smaller than the diffusion length of the carriers. As for the piezoelectric field, two factors can lead to the suppression. The first one is the effect of strain relaxation, similar to that reported for nanocolumnar $\mathrm{GaN} / \mathrm{Al}_{x} \mathrm{Ga}_{1-x} \mathrm{~N}$ quantum disks, ${ }^{10}$ where the pseudomorphic strain is effectively relaxed at the nanocolumn free surface at sidewall. Because the variations of the in-plane lattice parameter are distributed as a function of the depth from the sidewall surface, a future study will use a numerical simulation to analyze the spatial mapping of the band structure. The second factor is band bending at a surface, the degree of which changes depending on the Fermi level pinning. Such an effect is contributed from the charged surface states modified by the chemical nature of the surface.

Another interesting feature under high photoexcitation [Fig. 4(b)] is the appearance of sharp PL lines at 2.538 and $2.606 \mathrm{eV}$. Because the PL detection in this configuration is a cross sectional observation, the contribution from the $B$ and $C$ valence bands must be considered to identify each peak besides the $n=2$ conduction subband. The application of the polarization selection rule would be effective for further characterization. Nevertheless, the observation of sharp PL spectra from individual nanocolumns has allowed a detailed assessment of these interesting materials.

In conclusion, the detailed optical assessment of an InGaN/GaN nanocolumn reveals that the reduction of both the internal electric field and the exciton localization in this family should lead to promising properties as light emitting devices with sufficient oscillator strength.

The authors would like to thank to K. Ema, T. Sekine, and T. Ohtsuki at Sophia University for their valuable comments and discussion. This research was supported by CREST, Japan Science and Technology Agency.

${ }^{1}$ M. Yoshizawa, A. Kikuchi, M. Mori, N. Fujita, and K. Kishino, Jpn. J. Appl. Phys., Part 2 36, L459 (1997).

${ }^{2}$ A. Kikuchi, M. Kawai, M. Tada, and K. Kishino, Jpn. J. Appl. Phys., Part 2 43, L1524 (2004).

${ }^{3}$ M. Yoshizawa, A. Kikuchi, N. Fujita, K. Kushi, H. Sasamoto, and K. Kishino, J. Cryst. Growth 180/190, 138 (1998).

${ }^{4}$ S. Guha, N. Bojarczuk, M. Johnson, and J. Schetzina, Appl. Phys. Lett. 75, 463 (1999).

${ }^{5}$ E. Calleja, M. A. Sanchez-Garcia, F. J. Sanchez, F. Calle, F. B. Naranjo, E. Munoz, U. Jahn, and K. Ploog, Phys. Rev. B 62, 16826 (2000).

${ }^{6}$ K. Okamoto, K. Inoue, Y. Kawakami, M. Terazima, A. Tsujimura, I. Kidoguchi, and S. Fujita, Rev. Sci. Instrum. 74, 575 (2003).

${ }^{7}$ M. Sugawara, Phys. Rev. B 51, 10743 (1995).

${ }^{8}$ M. F. Shlesinger, Annu. Rev. Phys. Chem. 39, 269 (1988)

${ }^{9}$ Y. Kawakami, A. Kaneta, K. Omae, Y. Narukawa, and T. Mukai, in Progress in Nano-Electro-Optics, edited by M. Ohtsu (Springer, Berlin, 2005), Vol. IV, pp. 83-125.

${ }^{10}$ J. Ristic, C. Rivera, E. Calleja, S. Fernandez-Garrido, M. Povoloskyi, and A. D. Carlo, Phys. Rev. B 72, 085330 (2005). 